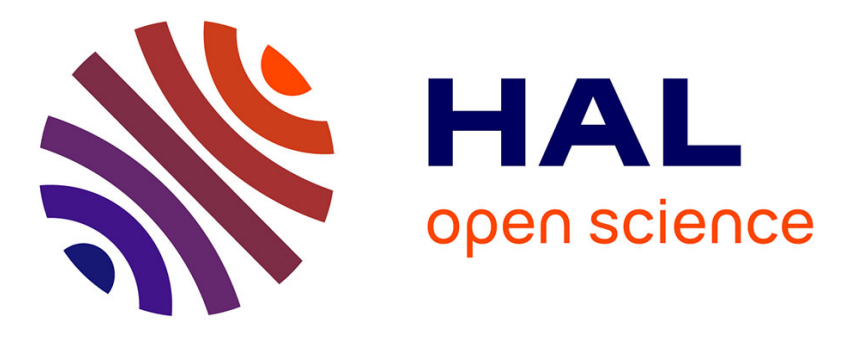

\title{
Video-to-Video for e-Health: Use Case, Concepts and Pilot Plan
}

\author{
Makis Stamatelatos, George Katsikas, Petros Makris, Nancy Alonistioti, \\ Serafeim Antonakis, Dimitrios Alonistiotis, Panagiotis Theodossiadis
}

\section{- To cite this version:}

Makis Stamatelatos, George Katsikas, Petros Makris, Nancy Alonistioti, Serafeim Antonakis, et al.. Video-to-Video for e-Health: Use Case, Concepts and Pilot Plan. 8th International Conference on Artificial Intelligence Applications and Innovations (AIAI), Sep 2012, Halkidiki, Greece. pp.311-321, 10.1007/978-3-642-33412-2_32. hal-01523101

\section{HAL Id: hal-01523101 \\ https://hal.science/hal-01523101}

Submitted on 16 May 2017

HAL is a multi-disciplinary open access archive for the deposit and dissemination of scientific research documents, whether they are published or not. The documents may come from teaching and research institutions in France or abroad, or from public or private research centers.
L'archive ouverte pluridisciplinaire HAL, est destinée au dépôt et à la diffusion de documents scientifiques de niveau recherche, publiés ou non, émanant des établissements d'enseignement et de recherche français ou étrangers, des laboratoires publics ou privés. 


\title{
Video-to-Video for e-Health: Use Case, Concepts and Pilot plan
}

\author{
Makis Stamatelatos ${ }^{1}$, George Katsikas ${ }^{1}$, Petros Makris ${ }^{1}$, Nancy Alonistioti ${ }^{1}$, \\ Serafeim Antonakis ${ }^{2}$, Dimitrios Alonistiotis ${ }^{2}$ and Panagiotis Theodossiadis ${ }^{2}$ \\ ${ }^{1}$ National and Kapodistrian University of Athens, Department of Informatic and \\ Telecommunications, Panepistimiopolis, Ilissia, Athens, Greece \\ ${ }^{2}$ National and Kapodistrian Medical School University of Athens, University General \\ Hospital Attikon, 1 Rimini Str. 12462 Haidari, Athens, Greece \\ \{makiss, katsikas, nancy\}@di.uoa.gr, petmakris@phys.uoa.gr \\ serafim.antonakis@gmail.com, dalonis@yahoo.gr, patheo@med.uoa.gr.
}

\begin{abstract}
Future Internet and smart cities are creating a very promising paradigm for providing advanced services to citizens. The paradigm of e-Health forms a valuable yet demanding use case for design, develop, deploy and provide related services. The aim of LiveCity project is to empower the citizens of a city to interact with each other in a more productive efficient and socially useful way by using high quality video-to-video $(\mathrm{v} 2 \mathrm{v})$; v2v can be used to improve medical services. This paper presents the related concepts, the scenario and the pilot set for the tele-monitoring service realization, deployment and provision.
\end{abstract}

Keywords: Video-to-Video, e-Health, Future Internet, Smart Cities

\section{Introduction}

Future Internet (FI) and Smart Cities are creating a very promising paradigm for unlocking multi-dimensional value propositions through exploring requirements constraints and delivery solutions: Cities are posing challenges; FI is promising solutions. Cities provide opportunities: real users, many users, structured users; Future Internet proposes real value: accessible, secure, safe.

From a business viewpoint a smart city is "a significant market of actors who share many needs in a complex, high-density and occasionally congested environment with a wealth of opportunities" [1]. From a services viewpoint, smart city citizens should enjoy ICT and FI enabled services realizing different needs; specifically in [2] six key smart cities' characteristics have been identified: (i) smart economy, (ii) smart people, (iii) smart governance, (iv) smart mobility, (v) smart environment, and, (vi) smart living. Obviously e-Health is one of the key challenges for smart living in smart city!

In [2] e-Health is defined as "patients and the public using Internet or other emedia to disseminate or provide access to health and lifestyle information or services." Therefore, e-Health is currently provided to citizens in emergency cases (e.g. tele-medicine), neither in large scale nor for "everyday" treatment. 


\section{Video to Video (V2V) Concept and Platform}

The aim of LiveCity project [3] is to empower the citizens of a city to interact with each other in a more productive, efficient and socially useful way by using high quality Video-to-Video (V2V) over the Internet. V2V can be used to save patients lives, improve city administration, reduce fuel costs, reduce carbon footprint, enhance education and improve city experiences for tourists and cultural consumers.

In this context, we are working on an e-Health framework promising to provide e-Health services to a wide range of scenarios including (i) real time "live" support to ambulance doctor from specialized emergency doctors as the ambulance is moving towards the hospitals, and (ii) everyday monitoring for patients with glaucoma in order to ensure conformance to identified treatment as well as provide patients with any additional information they need to know regarding treatment, progress or other issues "at home". V2V application services over high speed FI Infrastructures are the key means for the efficient provision of LiveCity e-Health Services.

V2V service as defined by LiveCity Project [3] is based on live high-definition interactive video communication easily available on user equipment. Video interactivity will be provided without complicated network provisioning or special video rooms: any video screen coupled with a video camera in any city should be able to connect a high-definition full screen video call at an attractive cost with seamless, utterly simple usability. Different requirements shall be taken into account for specific cases thus specializing platform capabilities towards realizing the scenario.

Today, live interactive high definition $\mathrm{v} 2 \mathrm{v}$ is well-supported in private networks owned by enterprises where certain QoS levels can be provided. However, smart cities need scalable solution and mass market: QoS is missing in the public internet.

An initial set of requirements and constraints have been already identified towards enabling V2V services, including among others:

- Inter-carrier interoperability and V2V service interoperability between carriers,

- Compatibility with most used UEs, simplicity of installation and usage,

- Seamless operation between end-point devices and applications,

- Globally reachable and accessible at reasonable cost,

- Ability to integrate additional features, (e.g. decision support system, or data pre-processing and inter-change).

Existing video platforms candidates for realizing V2V services (e.g. Skype, Oovoo) are not offering any facility to integrate additional features such as decision support system. Other open-source platforms (e.g. Jitsi, VLC, Ekiga) require assembly into platforms providing another alternative. In addition, tools are emerging on the Android platform for video support. As an initially estimate, best-of-breed of these tools can be used and applied to V2V devices (e.g. laptops, tablets, iPads, etc.) in order that they can use the "right-of-way". 


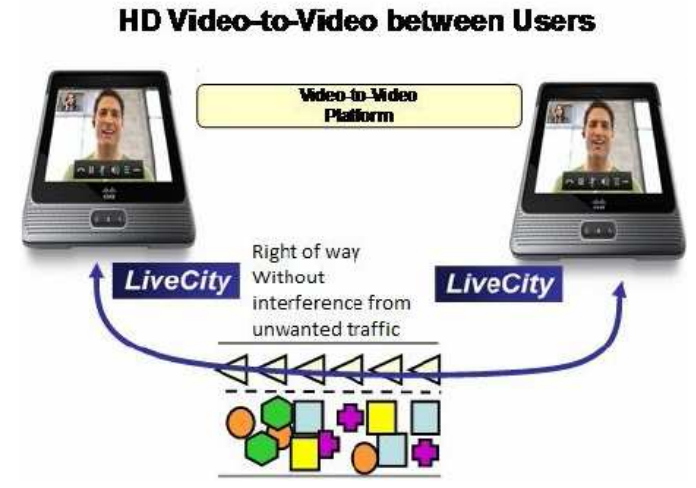

Fig. 1: V2V Devices using "Right of Way".

Fig. 2 depicts the architecture of the V2V platform as identified in the context of LiveCity Project. To obtain a v2v platform suitable for using existing V2V applications as well as security protocols, AAA and signaling protocols and applications should be integrated. Open source databases can provide login details. Security wrappers could provide security support and a content management system can be included to record the V2V. This is a very important feature for tele-medicine; content such as video, or/and video-shots can be part of patient's history and can be used for further reference or for more specialized treatment.

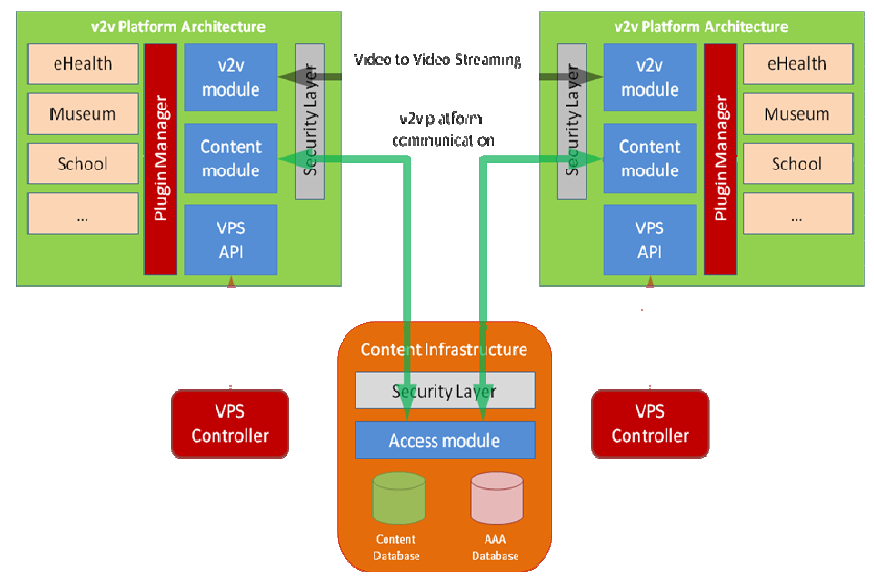

Fig. 2: V2V Platform Architecture.

\section{Scenario - e-Health: Tele-monitoring in patients}

This scenario of application of V2V services in e-Health establishes a link from a specialist doctor or other healthcare provider for monitoring the treatment of patients. This V2V approach will allow the GP and/or doctor specialist and/or nurse to have regular contact with the patient (while the latter being at his home) during patient's 
medical treatment. Patients' Telemonitoring use case is to be developed through a centre of excellence in surgical, clinical and experimental research areas comprising nine (9) specialized departments thus covering a number of areas of expertise, such as glaucoma, medical retina, vitreoretinal surgery, corneal transplantation, cataract surgery, refractive surgery.

The involved University Clinic [4] treats patients with glaucoma from all over Attica region and Greece, that have to commute to the hospital for checking wellapplying of the treatment; in this sense, the $\mathrm{v} 2 \mathrm{v}$ service will ensure conformance of treatment, the correct application of the eye-drops as well as access to medical information regarding questions or queries which may arise with regards to the treatment being applied. In the scope of the proposed activities, doctors and/or other medical staff will realize appropriate V2V connections with patients and will monitor them, in order to have a proper patients' control during patients' medical treatment. The high quality of live $\mathrm{V} 2 \mathrm{~V}$ will eliminate the necessity for the patients to travel and visit the hospital, where appropriate. Fig. 3 depicts the considered layout.

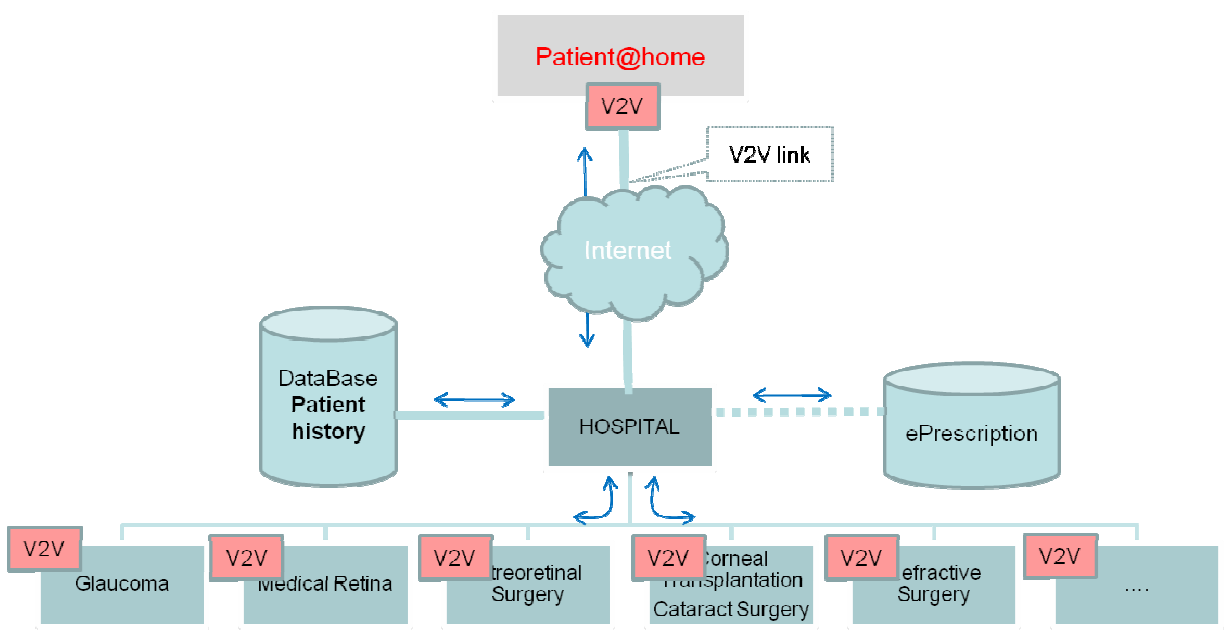

Fig. 3:V2V services for e-Health - Patient Monitoring.

The "User" of this Use Case (the patient) is provided with V2V service at home as a means for "every-day" monitoring and treatment; this service is also applicable in case that the patient has been planned for a surgery or has passed surgery. Patient is provided with the needed infrastructure for the V2V service (e.g. a digital camera and a laptop for deploying the $\mathrm{V} 2 \mathrm{~V}$ client). $\mathrm{V} 2 \mathrm{~V}$ service can be provided either by the hospital or a network service provider. The patient has been provided with a highspeed internet connection.

The main value provider in this scenario stands for the Hospital hosting the V2V e-Health service. The hospital may have established a number of specialized departments providing specific e-Health services; in the figure, the Hospital is hosting a number of ophthalmology departments. A central management unit is located in the hospital aiming at authenticating the patient and directing him to the appropriate department. The hospital may have access to each patient history which may be 
provided by the public sector; otherwise local database may be maintained by the hospital. Moreover, for the treatment part of the use case, access may be needed to the e-prescription database in order to prevent conflicts.

\subsection{Use Case: Glaucoma Treatment}

Most treatments for glaucoma are designed to lower and/or control intraocular pressure (IOP), which can damage the optic nerve that transmits visual information to the brain. Glaucoma eye-drops often are the first choice over glaucoma surgery and can be very effective at controlling IOP to prevent eye damage and loss of vision.

A newly diagnosed person with glaucoma may need to have his eye pressure checked every week or month until it is under control. Even when his eye pressure is at a safe level, he may need to see his doctor several times a year for checkups.

For the doctors and the Hospital it is important that the doctor listens and responds to patients' concerns and questions and is available for calls and checkups; a good working relationship between patient and eye doctor is the key to effective glaucoma treatment. It is also important to ensure that patients apply properly the eye drops in their eyes. Proper use of glaucoma medication can improve the medicine's effectiveness and reduce the risk of side effects.

For patients, it is substantial to take their medications regularly and exactly as prescribed if they are to control their eye pressure. Unless eye drops are used correctly, they will not be effective and glaucoma can worsen: Almost one half of all glaucoma patients fail to take their eye drops correctly: For some, this means forgetting one or more doses of an eye drop; others may remember to take them, but do so incorrectly. In this case, the eye drop may not be placed properly on the eye or may be washed out by excessive blinking or tears. The instructions-steps that a patient must follow are:

- "Wash your hands, and hold the bottle upside down”,

- "Tilt your head back, hold the bottle in one hand and place it as close as possible to the eye",

- "With the other hand, pull down your lower eyelid",

- "Place the prescribed number of drops into the lower eyelid pocket. If you are using more than one eyedrop, be sure to wait at least 5 minutes before applying the second eyedrop",

- "Close your eye or press the lower lid lightly with your finger for at least one minute to keep the drops in the eye and help prevent the drops from draining into the tear duct, which can increase your risk of side effects".

Taking an eye drop at the wrong time is also incorrect, and can lead to worsening of glaucoma. If the interval between taking the same eye drop is either too short or too long, its effectiveness will be reduced. For example, an eye drop which is taken too close to another may actually wash the first one from the outside of the eye and reduce its effectiveness. Using more than one eye drop for each eye is another way in which eye drops often are used incorrectly. Some patients are under the mistaken impression that two or more drops at a time may be more effective than one. In fact, if 
a single eye drop is administered correctly, then using more than one may cause a drug overdose with consequent side effects.

It is easily understood that V2V tele-monitoring would be a very useful tool for medical practitioners. It will help to reduce glaucoma patients' visits to the hospital, ensures proper use of eye drop and could be a good reminder for patients.

\subsection{A Business View}

Fig. 4 presents a business view on the tele-medicine scenario as an initial model: the roles and revenue flows based on working assumptions for the service provision. Such work will be further elaborated in order to provide hints on the feasibility of tele-monitoring scenario as "value proposition".

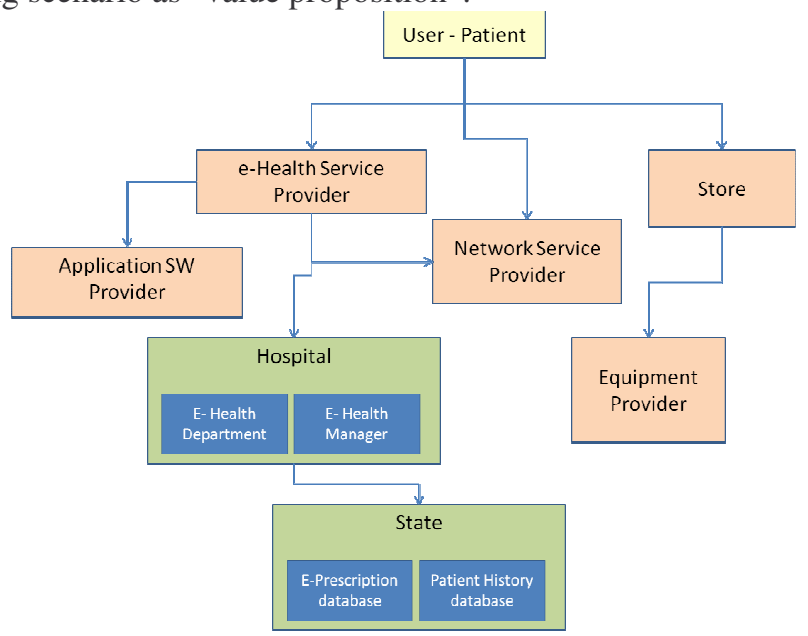

Fig. 4: A Business View for Telemedicine Scenario

User - Patient is provided with e-Health service and pays corresponding fees and costs for the service client. He moreover pays the Network Service Provider for connectivity and the Store for acquiring the needed equipment. E-Health Service Provider provides the service and pays the Hospital for hosting the service, the Network Service Provider for connectivity, and the Application SW Provider for developing the application. Store sells "ready to use" equipment and pays the Equipment Provider. Network Service Provider provides the needed infrastructure and resources for the communication between the user and e-health service. Application SW Provider develops the applications for the use case. Equipment Provider develops hardware devices. Hospital is hosting the e-Health Service in terms of scientific support; hospital is the actual implementer of the e-Health Service but is separated from the e-Health Service Provider to model the more generic case in which the service is provided by, for example, an insurance agency, an enterprise etc. Hospital may need to pay some share revenue with the State. State maintains knowledge bases (e-prescription and patient history) which may be valuable for clarity and completeness of the use case. 


\section{Scenario realization}

\subsection{Network Architecture}

Generally, 4G wireless technology (LTE and WiMAX) will be utilized for the $\mathrm{V} 2 \mathrm{~V}$ scenarios realization coupled with $3.5 \mathrm{G}$ wireless technology where $4 \mathrm{G}$ is not available and also using fixed line xDSL broadband. Key target is the logical technical architecture and implementation of the necessary ingredients to support Future Internet V2V services and "right of way" technology on public Internet infrastructure.

The involved parties (Fig. 5) are placed according to the following requirements. First of all, the patients' access at the V2V service can be twofold. The former is a fixed and low-cost xDSL connection for covering the needs of those who are remotely treated by their homes (e.g. post-surgical control), while the latter is a high quality wireless/cellular link (WiFi or 3.5/4 G) using the network operators' respective infrastructure (e.g. the WLAN/WiMAX stations depicted in Fig. 5). The wireless access is provided in order to cover the needs of the patients' that cannot access the service otherwise. For this reason several PoPs (e.g. public hot-spots) should be available by the network provider so as to reassure an always-available service and take care of several emergency situations. In addition, at the e-health provider's side, a high quality and bandwidth line should be established with the network provider for guaranteeing the seamless provision of the e-health services and providing an excellent quality of experience to the medical personnel. Such requirements are critical for the maximization of both the patients' and the medical stuff's satisfaction.

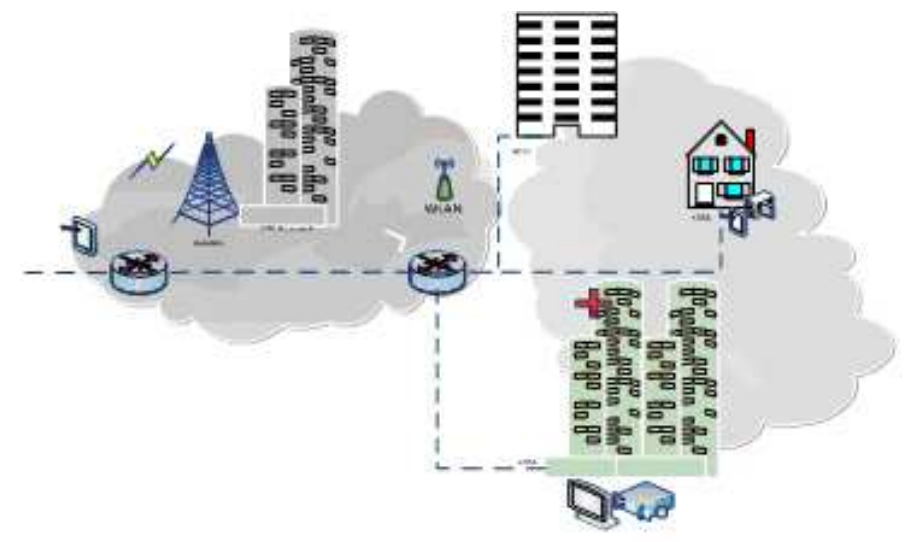

Fig. 5: Network layout for Telemedicine Scenario.

The involved parties, as depicted in the above architecture, are placed according to the following requirements. First of all, the patients' access at the V2V service can be twofold. The former is a fixed and low-cost xDSL connection for covering the needs of those who are remotely treated by their homes (e.g. post-surgical control), while the latter is a high quality wireless/cellular link (WiFi or 3.5/4 G) using the network 
operators' respective infrastructure (e.g. the WLAN/WiMAX stations depicted in Fig. 5). The wireless access is provided in order to cover the needs of the patients' that cannot access the service otherwise. For this reason several PoPs (e.g. public hotspots) should be available by the network provider so as to reassure an alwaysavailable service and take care of several emergency situations. In addition, at the ehealth provider's side, a high quality and bandwidth line should be established with the network provider for guaranteeing the seamless provision of the e-health services and providing an excellent quality of experience to the medical personnel. Such requirements are critical for the maximization of both the patients' and the medical stuff's satisfaction.

\subsection{Network Requirements}

The network requirements are referred mainly to the bandwidth needs of the high quality, real-time, video to video streaming platform as well as the identification and configuration of measurement points for the KPIs collection.

High quality video streaming requires symmetrical high bandwidth wired or wireless connections that may guarantee a bandwidth of $10 \mathrm{Mbps}$ (Streaming of HD video). The network technologies depicted in Fig. 5, (i.e. xDSL, Wi-Fi, WiMAX, 3.5G and $4 \mathrm{G}$ LTE ) are used to ensure the provision of the service along with the VPS "right of way" technology that required QoS levels.

\subsection{Setting the Pilot}

The procedure for setting and evaluating the pilot has been planned as follows:

- Set the facilities and the corresponding system requirements,

- Elaborate on the pilot scenarios,

- Identify needed results to be obtained,

- Set simulations,

- Identify KPIs and their correlation with the network performance,

- Align results obtained from the pilots to the KPI requirements,

- Collect and analyze the measurements gathered by the V2V Platform.

The facilities for the realization of the aforementioned scenario should be provided by a network operator. More specifically, an IP-based xDSL testbed should comprise connections of patients directly to the Hospital, under appropriate terms and/or conditions, in the scope of the cooperation between the operator and the healthcare provider. Additional xDSL connections should be also established between the healthcare provider and the service provider as well as connectivity to the doctors unit.

The essential need of the scenario case is remote monitoring and inspection of typical every-day treatment procedures that must be followed by the patients; this can be the use of eye drops (as presented in section 3.1). In that case a typical video camera could be used (i.e. build-in cameras of tablet devices). In other to monitor 
processes where a clean and robust picture of the human eye is required more sophisticated camera/video recording equipment should be present. Photos and video using a typical tablet's camera cannot provide a clean and usable (by a doctor) eye picture (i.e. the picture can be confusing).

This occurs due to technical limitations of a device's hardware (low quality lens, lighting issues). To support extra and more advanced monitoring processes in order to provide a picture/video of a patient's eye at the required quality an external camera with advanced lens system could be used. Adding external hardware and custom circuitry to a platform increases complexity by all aspects. This requirement consequently raises the restrictions and limitations of the overall hardware configuration.

External USB devices and specifically the UVC compliant cameras might be difficult to integrate with Android tablets. Moreover, usage of external hardware might require some extra information to be communicated between the service and client point such as the focus and zoom controls of an advanced external camera lens system which should be remotely adjusted. This might require extra than the usually available connectivity of a tablet device (USB ports).

Since a technical goal of this use case is to enable a high quality v2v link between endpoints (service point, client point) and keeping in mind that high quality video means High Definition (Quality 720p which means at least 1200x720 pixels video resolution) the suggested application layout for the telemedicine use case scenario requires full screen video projection. Any user interface layout that reduces video size results to a down scale procedure which invalidates the primary project goal. User controls (pause/hold/video mute/audio mute/screenshot and potentially audio or video record) could be overlaid and semi-transparent over the video.

The list below summarizes the mentioned aspects in terms of system requirements:

- High performance CPU enabling processing and manipulation of high resolution video (Dual Core $1 \mathrm{GHz}$ CPU),

- High quality integrated cameras (at least 3.0 MP),

- High resolution display that enables play back-of high definition video (800 x 1280 pixels@10 inches),

- USB Host support that allows connectivity of external hardware. This strong requirement has not been confirmed that is provided by android tablets, such as:

- Samsung Galaxy Tab II P3100 3G,

- Samsung Galaxy Tab 2 10.1 P5100,

- X86 Compatible tablets (Usage of Linux OS),

- Acer Iconia TAB 500.

With regard to the realization of the proposed pilot action, the subsequent hospital should provide the required facilities (e.g. a clinic or hospital ward). Towards this direction, the appropriate IT-technical and non-technical setup for the scenario should be prepared and the needed medical personnel have to be provided in order to fulfill the tasks which aim to be achieved by the related scenario. Some indicative technical requirements for this scope would be the installation of terminal stations, IP cameras and microphones to the patients' and doctors' rooms as well as the personnel (doctors, 
medical staff) training for facilitating the familiarization with the new technological requirements.

At the service provisioning side, the essential aim will be to describe, analyze and assess results to be obtained by the entire telemonitoring scenario, via the inclusion of $\mathrm{V} 2 \mathrm{~V}$ facility in the communication between the healthcare provider (doctors in the hospital) and the patients. To this end, various monitoring and communication capabilities should be supported for the efficient remote control of service parameters such as the provided bandwidth, PER, delay and jitter. In order to meet the strict requirements of a modern $\mathrm{V} 2 \mathrm{~V}$ application, one should highly consider reassuring the QoS levels of the multimedia services. To this end, the dedicated links among the health-care provider and the patients' side should be established as VPNs by the network service provider, so as to guarantee the high level provision of the aforementioned KPIs.

Considering the patients' side regarding the perceived QoE, several quality of life measures should be collected using client applications. Such measures could be related to the treatment satisfaction of the patient and the wellbeing score. The latter process is considered highly useful for the effective feedback of the whole application, since, by aligning the obtained results (e.g. patient satisfaction) together with the KPI requirements (e.g. delay, jitter, PER), the perceived QoE can be significantly improved.

Finally, the massive data gathering procedure of the V2V service platform can be also exploited using several data analysis mechanisms in order to identify the characteristics of the service and take advantage of the correlations.

\section{Conclusion - Future Work}

In this paper, we presented the LiveCity approach on patient's tele-monitoring. Starting with smart cities and future internet we set the need and scope of our planned work: the $\mathrm{v} 2 \mathrm{v}$ platform and service. Then we provided a detailed description of the e-Health scenario featuring tele-monitoring of patients focusing on glaucoma every-day treatment "from home". We described the scenario layout as well as a business view of the scenario. In the context of presenting the scenario realization, we detailed the envisaged network architecture and we elaborated on a set of system requirements towards setting the pilot; a number of steps have been also identified and analysed accordingly.

The next immediate steps are focusing on defining system and functional requirements for the deployment of tele-monitoring scenario at the v2v platform, the identification of the target patients group as well as the network and service infrastructure realization.

\section{Acknowledgment}

This work has been performed in the framework of the ICT- PSP 297291 LiveCity Project funded by the European Commission. 


\section{References}

1. Future Internet for "Smarter Cities" and User-centric Open Innovation: http://www.future-

internet.eu/fileadmin/documents/ghent_documents/Session_VI_Smart_Cities/FI_for_ Smarter_Cities_Borjeson.pdf

2. Smart cities Ranking of European medium-sized cities: http://www.smartcities.eu/download/smart_cities_final_report.pdf

3. FP CIP LiveCity Project, http://www.livecity-psp.eu/

4. The Attikon Hospital, http://www.attikonhospital.gr/ 